\title{
Research Paper \\ Creating a Lip-reading Training Program and Examining its Effect on Speech Recognition in Men Aged 18-25 With Normal Hearing
}

\section{Seyede Faezeh Fazelian ${ }^{1} \odot$, Ali Mohammadzadeh² ${ }^{*}$ Homa Zarinkub² $^{2}$, Alireza Akbarzadeh Baghban ${ }^{3}$}

1. Department of Audiology, School of Rehabilitation, Hamadan University of Medical Sciences, Hamadan, Iran.

2. Department of Audiology, School of Rehabilitation, Shahid Beheshti University of Medical Sciences, Tehran, Iran.

3. Department of Basic Sciences, School of Rehabilitation Sciences, Shahid Beheshti University of Medical Sciences, Tehran, Iran.

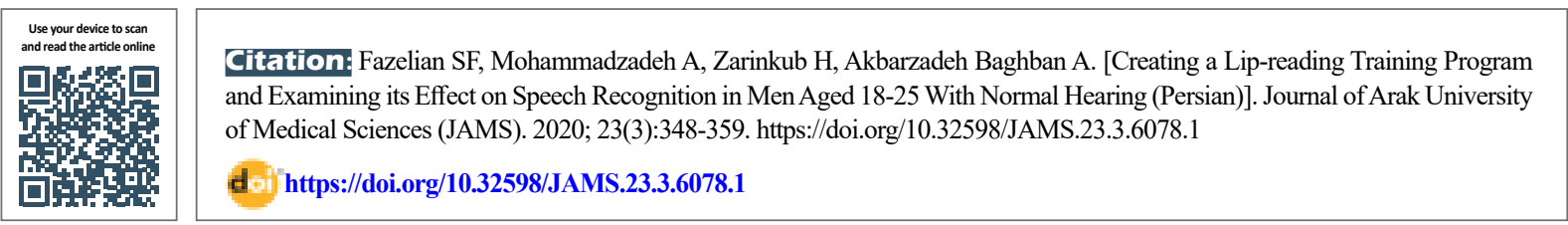

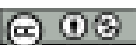

Article Info:

Received: 20 Jan 2020

Accepted: 04 Jun 2020

Available Online: 01 Aug 2020

Key words:

Lip reading, Speech recognition, Sarah lip reading test

\section{ABSTRACT}

Background and Aim Lip reading is extraction of speech data from the activity of the lower part of face, particularly jaws, lips, tongue, and teeth that is a natural skill in people with hearing loss. The main purpose of lip reading is to increase the independence of people with hearing loss. Looking at the speakers facial movements significantly increase the ability to understand the spoken words, particularly in the environments where noise is present. In fact this reflects the important role of visual signals. In current study we consider the effects of education on lip reading ability by planning and instructing a lip reading program in male subjects.

Methods \& Materials Sara lip reading test 1 and 2 were used for the assessment of lip reading skills and for studying the effects of lip reading training on recognition of speech. This intervention as a semi-experimental study, was conducted on 27 male students, selected by available sampling. For data analysis, statistical analysis of variance with repeated measurements and Mcnemar was used.

Ethical Considerations This research with the code IR.SBMU.REC.1394.144 has been approved by the ethics committee of Shahid Beheshti University of Medical Sciences.

Results There were significant differences between Sara lip reading test 1 scores in 2 positions $(P=0.000)$. Lip reading test scores also showed significant differences in Sara lip reading test 2 in 2 positions $(P=0.000)$. Also between the scores of Consonant-vowel, consonant-vowel syllables, in the position before teaching lip-reading and then, was significant difference $(P<0.05)$.

Conclusion The findings of this study showed that instructing lip reading has a positive effect on lipreading ability in people.

\section{Extended Abstract}

\section{Introduction}

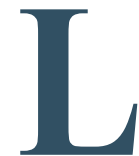

ip reading is the extraction of speech data from the activity of the lower part of the face, especially the jaws, lips, tongue and teeth, which is a natural skill in hearing people. The main purpose of lip reading is to in- crease the independence of people with hearing impairments. Recent experience has shown that seeing the speaker's lips increases sensitivity to acoustic information and decreases the speech detection threshold in the presence of noise. The experience of the person as a listener shows that spoken sounds are heard louder when looking at the speaker $[1,2]$.

Obtaining visual information from the speaker's mouth and face movements play an important role in understanding spo-

\section{* Corresponding Author:}

Ali Mohammadzadeh, PhD.

Address: Department of Audiology, School of Rehabilitation, Shahid Beheshti University of Medical Sciences, Tehran, Iran.

Tel: +98 (912) 1876285

E-mail: amzadeh@sbmu.ac.ir 
ken language [3-5]. Also, people who, for various reasons, their speech communication ability is partially or completely impaired, use this supplementary information $[6,7]$. In the present study, by designing and constructing a lip-reading training program, we investigated the effect of training on lipreading skills of 18 to 25 -year-olds.

\section{Materials and Methods}

This intervention study was performed before and after lip reading training. The study population consisted of 27 male students aged 18-25 years with a Mean \pm SD age of $20.67 \pm 2.28$, who participated voluntarily from the rehabilitation faculties of Shahid Beheshti University of Medical Sciences and Hamedan University. First, a questionnaire including personal and medical information of individuals as well as written consent was completed to check the inclusion criteria. Right-handedness, monolingualism, lack of hearing and vision impairment were considered as the inclusion criteria.

Sara 1 and Sara 2 lip reading tests were used to evaluate lip reading skills and to evaluate the effect of lip reading training on speech recognition of the samples in the study. For data analysis, repeated measures ANOVA and McNemar tests were used. This study consisted of two parts: the first part was the design and construction of a lip-reading training program, which included 7 stages, and the second part, included the implementation of tests and the use of the program.

\section{Results}

In the present study, all subjects (27 participants) were evaluated by both Sara 1 and Sara 2 lip reading tests. In statistical analysis with repeated measures ANOVA, there was a significant difference between Sara 1 test lip reading scores before and after training $(\mathrm{P}=0.000)$. Also, in statistical analysis to analyze the results of Sarah 2 lip reading test, a significant difference was observed between the scores of this test before and after training $(\mathrm{P}=0.000)$. In Table 1 , statistical indicators related to Sara 1 and Sara 2 lip reading scores are reported in two situations. In order to investigate the effect of lip reading training, McNemar statistical analysis was used to compare the "consonant-vowel, consonant-vowel" (cv-cv) syllable scores before and after training (Figure 1 and 2). Out of 23 cv-cv syllables, 4 syllables showed a significant difference $(\mathrm{P}<0.05)$.

Also, in the content of Sara 1 lip reading test, which was used in a total of 20 sentences, there were 176 vowels, which in the review of vowels a significant difference between the scores before and after training in each of the 6 vowels $(/ \mathrm{a} /$, /e/, /i/, /â/, /o/, /u/ was observed ( $\mathrm{P}=0.000)$.

\section{Discussion}

Comparing the scores of Sara 1 lip reading test before and after training, a significant difference was observed in the

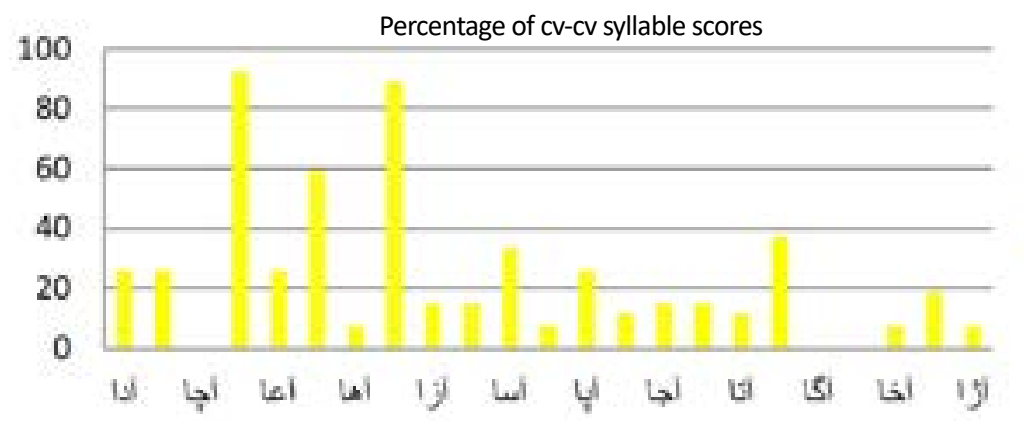

Male

Figure 1. Percentage of consonant-vowel, consonant-vowel syllable scores before training

Table 1. Statistical indicators of lip reading scores of Sara 1 and Sara 2 tests before and after training

\begin{tabular}{ccccc}
\hline Variables & Quantity & Min & Max & Mean \pm SD \\
\hline Sarah 1 lip reading test score before training & 27 & 0 & 50 & $15.19 \pm 2.52$ \\
\hline Sarah 1 lip reading test score after training & 27 & 5 & 80 & $42.41 \pm 4.01$ \\
\hline Sarah 2 lip reading test score before training & 27 & 12.9 & 51.6 & $23.41 \pm 1.61$ \\
\hline Sarah 2 lip reading test score after training & 27 & 17.2 & 51.6 & $35.51 \pm 1.68$ \\
\hline
\end{tabular}




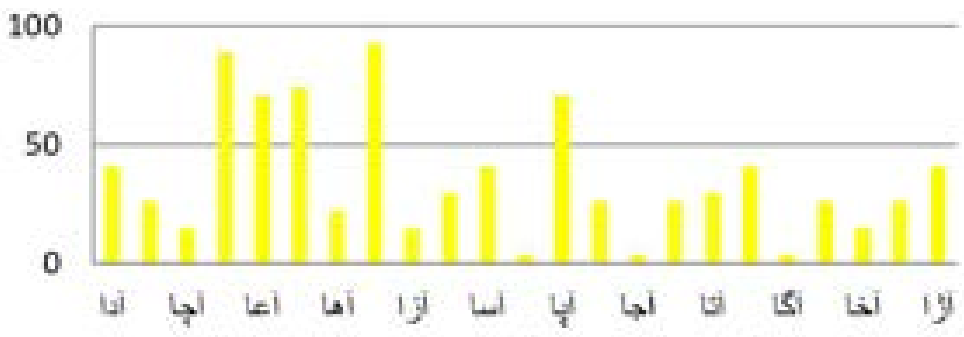

Figure 2. Percentage of consonant-vowel, consonant-vowel syllable scores after training

\section{Male}

Journal of Arak University of Medical Sciences scores $(\mathrm{P}=0.000)$ which indicated the positive effect of lip training on speech recognition and efficiency of lip training program. The Mean percentage of "speech recognition by lip-reading" scores was 15.19 before training, while this Mean was 42.41 after training. Improving the scores showed the positive effect of training on lip reading and improving speech recognition skills. This results was consistent with the results of studies that have measured the effect of training on lip reading [16-19].

There are conflicting results about lip reading training; in fact, there are differences between short-term and long-term training, individual and group training, and training for children and the elderly. This sensory process appears to have limitations, at least in the visual system, although its nature is unclear. Some people may not improve on speech perception tests, but if they are asked if they have benefited from lip reading training, they would talk for hours in support of lip-reading! $[12,19]$.

\section{Conclusion}

A noteworthy point in the results of the present study was that most people acknowledged that when watching the lip reading training program and especially the filmed videos (seeing and hearing the pronunciation of syllables), their subconscious mind simultaneously repeated that phoneme or syllable so that they could have a better learning experience. This point can be justified by the motor theory of speech perception. According to this theory, speech is produced by the same process that is perceived.

According to the results of the present study, lip reading training is one of the factors that improves lip reading performance and subsequently improves speech recognition. According to this result, the lip reading training program could have a positive effect on lip-reading performance. The educational content used in this program can be used to educate people, especially hearing impaired people, either as personal use at home or as group training in rehabilitation programs.

\section{Ethical Considerations}

\section{Compliance with ethical guidelines}

Gaining informed consent and providing the results were ethical principles in this study. This research with the code IR.SBMU.REC.1394.144 has been approved by the ethics committee of Shahid Beheshti University of Medical Sciences.

\section{Funding}

$? ?$

\section{Authors' contributions}

All authors contributed to the preparation of this article.

\section{Conflicts of interest}

The authors declared no conflict of interest.

\section{Acknowledgements}

The authors consider it necessary to thank the assistance of the Physiotherapy Research Center, the Vice Chancellor for Research and Technology of Shahid Beheshti University of Medical Sciences, the Department of Audiology of the Faculty of Rehabilitation of Shahid Beheshti University of Medical Sciences and all students participating in this study. The cooperation of Dr. Gita Movallali is also appreciated. 


\title{
ساخت برنامه أموزشى لبخوانى و بررسى اثر آن بر بازشناسى تَفتار افراد A (تاه اساله با شنوايى

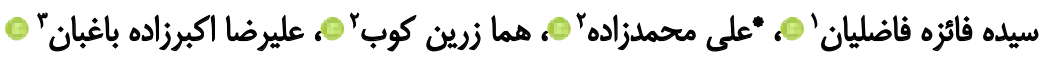 \\ ا I. كروه شنوايى شناسى، دانشكده علوم توانبخشى، دانشكاه علوم يزشكى همدان، همدان، ايران.

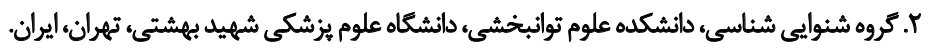

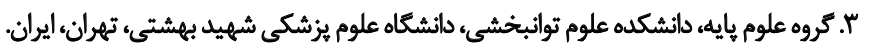

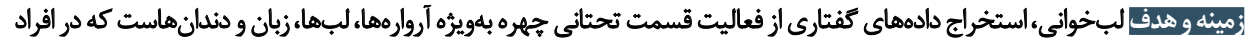

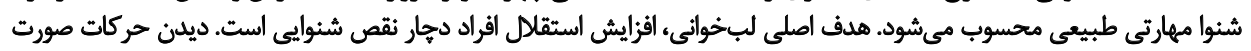

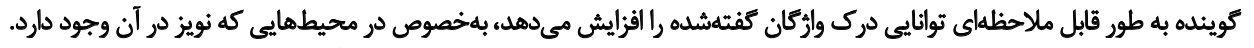

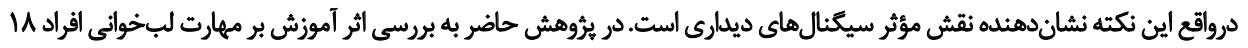

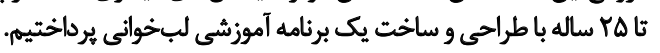

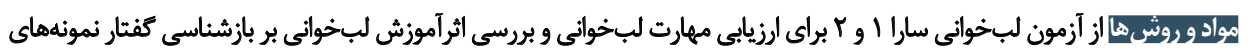

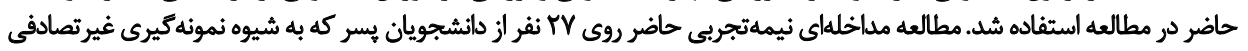

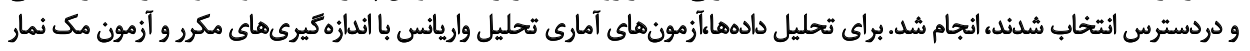
مورداستفاده قرار كرفتند.

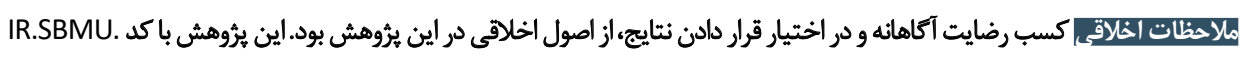

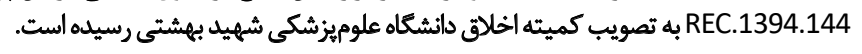

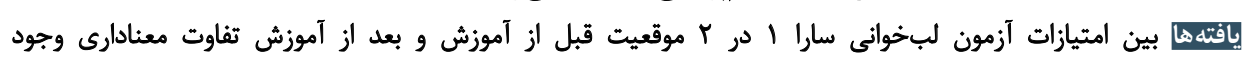

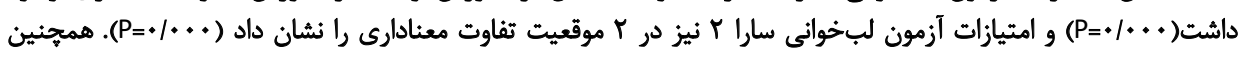

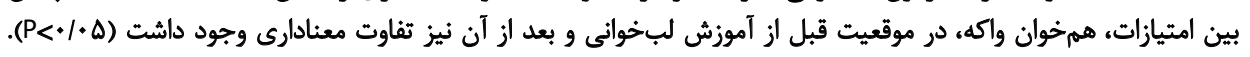

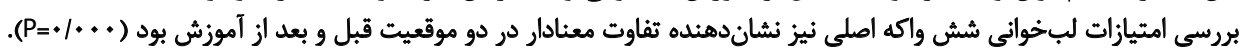

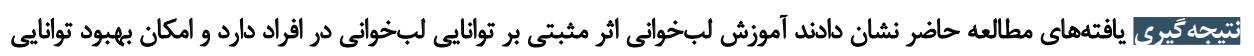

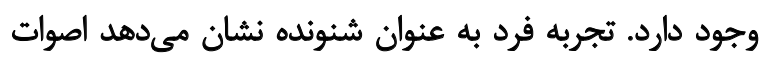

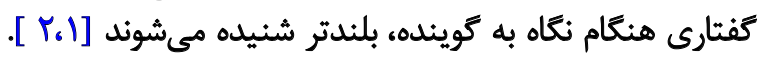

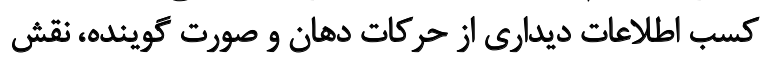

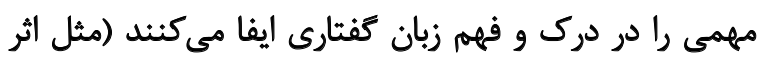

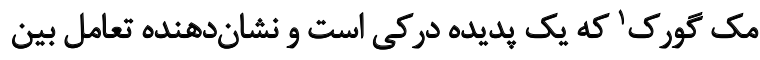

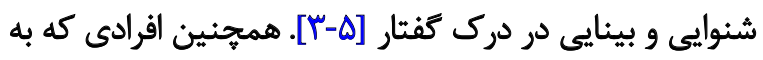

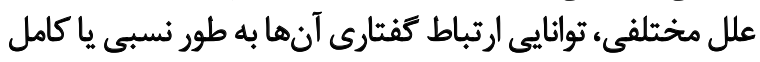

لبخوانى به توانايى فهم نسبى كفتار با نكاه كردن بر لبهان

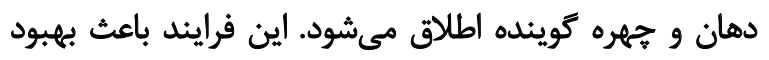

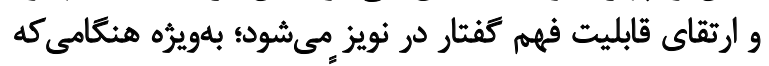

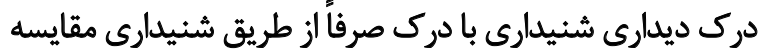

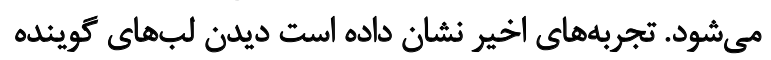

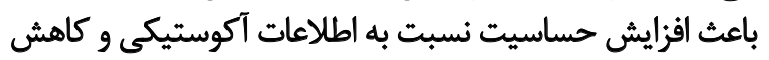

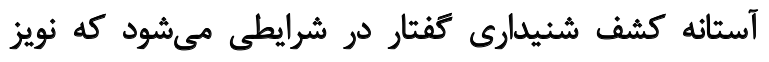

ㄷ..… 
برنامه آموزشى مولر ويلز را طراحى كرد. روشهاى تهي آموزش

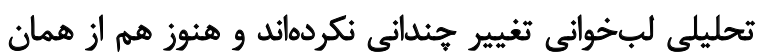

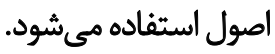

مربيانى كه از اين روش استفاده مى كنئد فعاليتهاى تمرينى

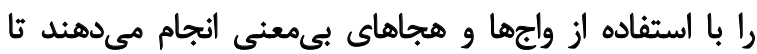

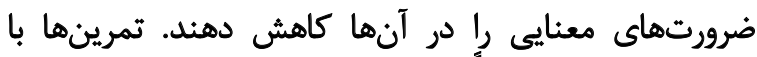

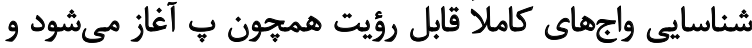

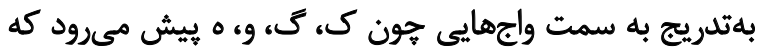

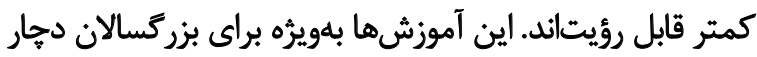

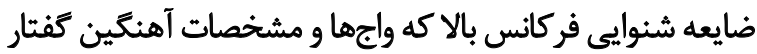
را تشخيص مى دهند بسيار مفيد است.

روش ديكر، روش تركيبى است كه در آن از جملات، عبارات

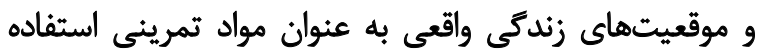

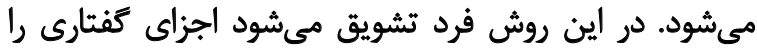

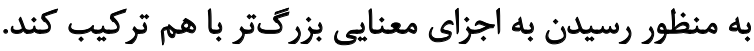

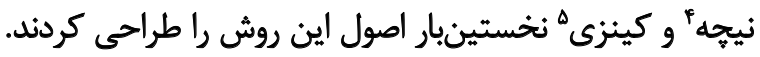

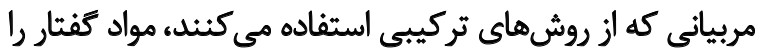

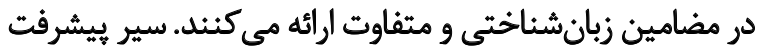

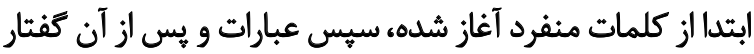

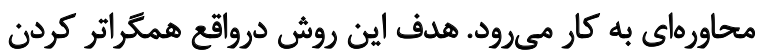

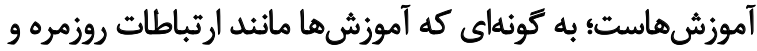

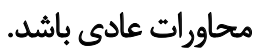

بهترين شيوه آموزش، اين است كه آن را همراه باصدا آموزش

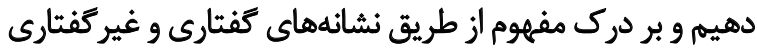

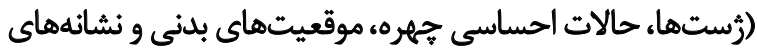

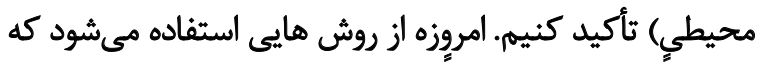

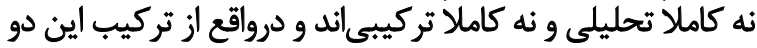

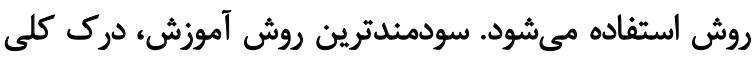

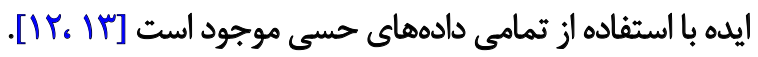

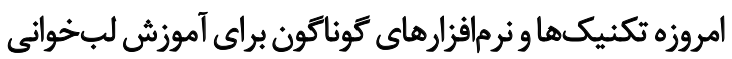

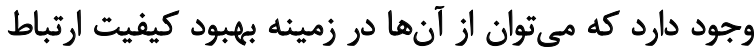

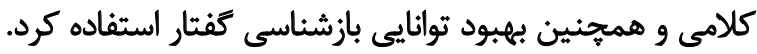

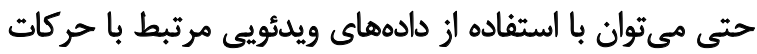

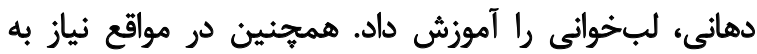

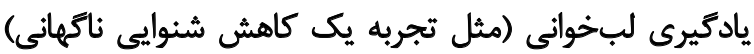

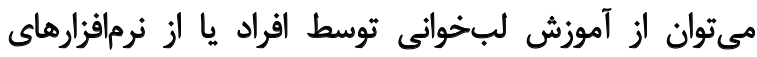

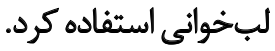

از آنجايي كه تاكنون در ايران مطالعهايى مبنى بر تأثير آموزش آثراني

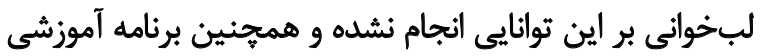

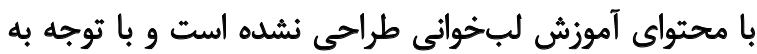

دجار اختلال شده است، از اين اطلاعات مكمل استفاده مي كنيند

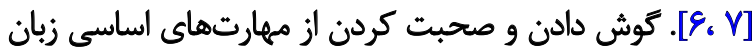

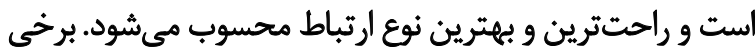

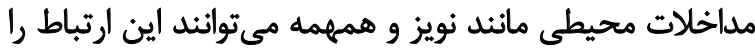

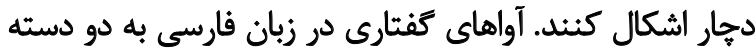

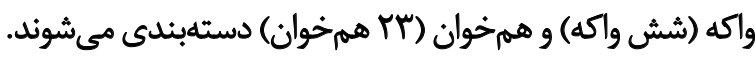
مطالعات انجامشده روى درك ويرّكى هاي خاص كَفتار در

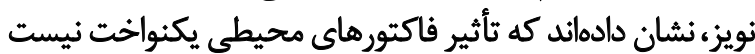

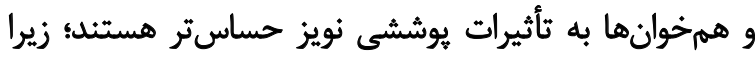

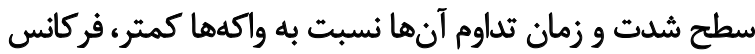

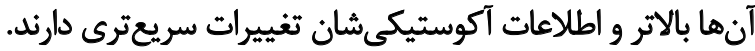

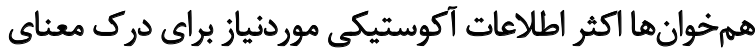

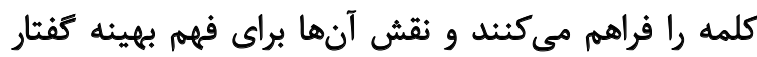

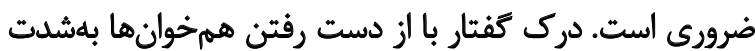

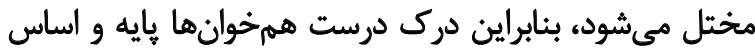

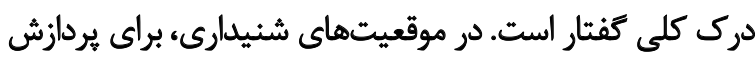

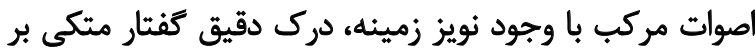

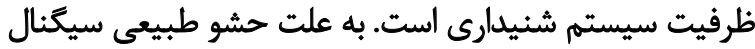

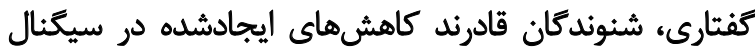

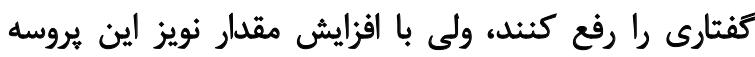

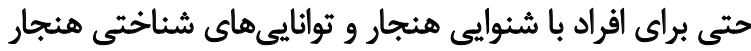

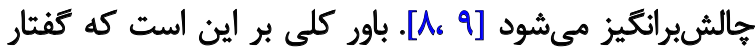

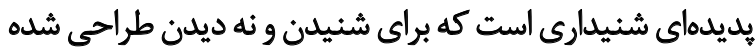

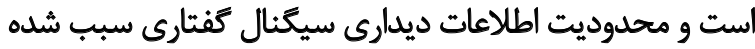

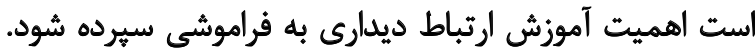

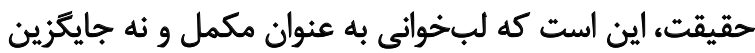

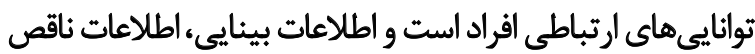

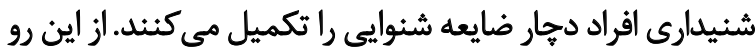

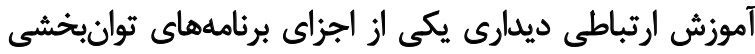

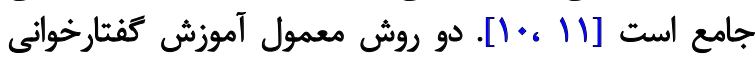
(لبخوانى) عبارتاند از: روش تحليلى و روش تركيبى.

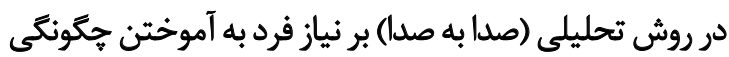

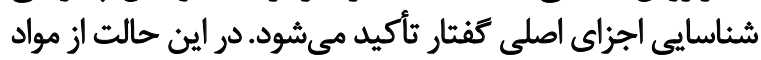

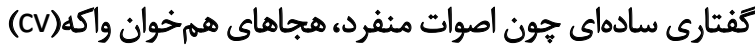

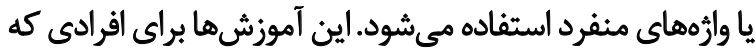

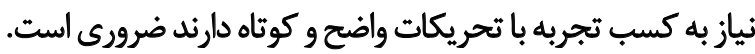

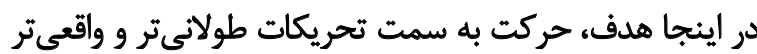

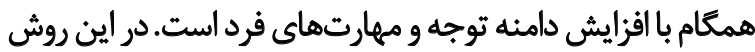

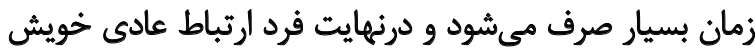

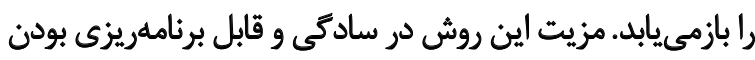

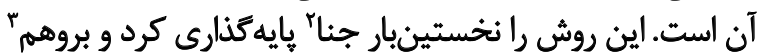


مى سويم. تصويربردارى با استفاده از دوربين فيلمبردارى و عكاسى

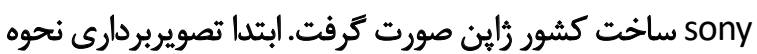

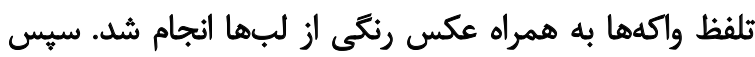

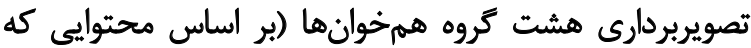

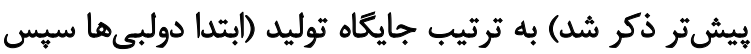

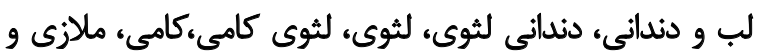

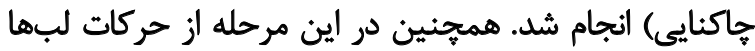

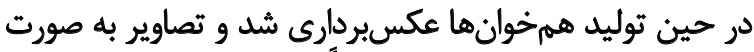

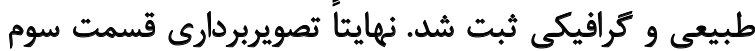
يعنى كفتار بيوسته انجام شد.

9. تدوين و تنظيم فايلهاى تصويربردارىشده با استفاده از

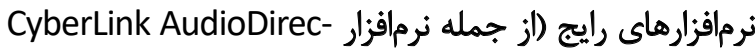

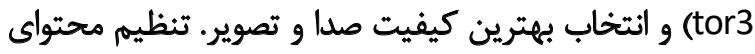

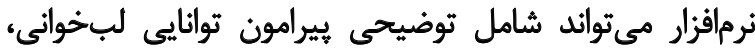

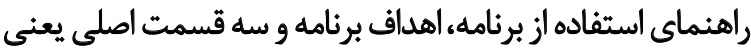
واكهها، همخوانهاو كفيتار يُ ييوسته باشد.

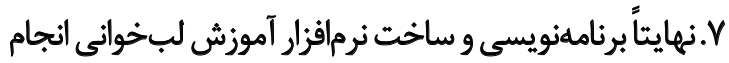
شد.

ب)بخش دوم، شامل اجراى آزمونها و بهكاركيرى برنامه

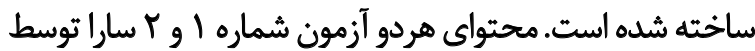

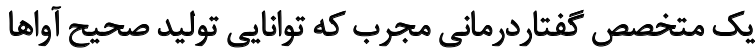

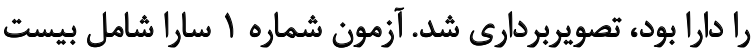

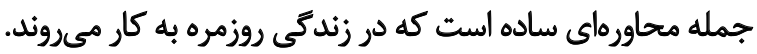

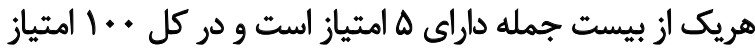

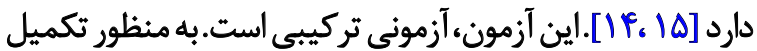

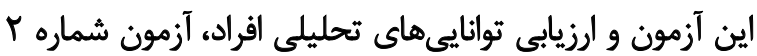

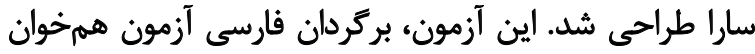

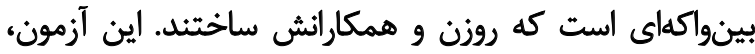

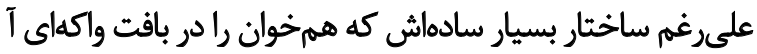

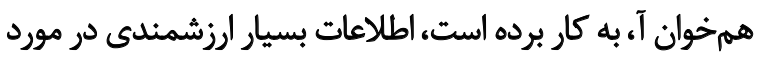

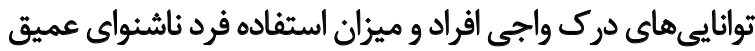

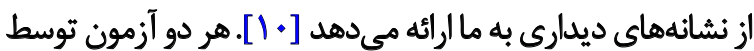
خانم دكتر موللى طراحى شده اند.

\section{شرايطل الهراى آزّمون}

ارائه آزمون به صورت فيلم ويدئويى و تصاوير (با هدف يكسان

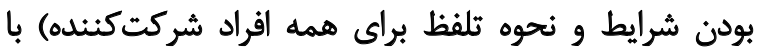

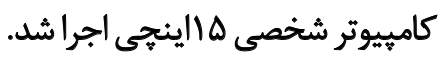

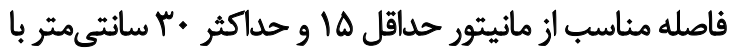

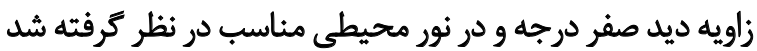

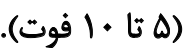

اجراى آزمون به اين ترثيب انجام شد كه فرد در يك اثاق با
جايكاه مهم لبخوانى در بهبود بازشناسي كَفتار واثر مثبت آن در

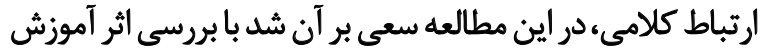

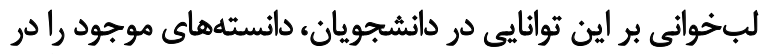

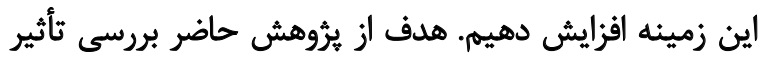

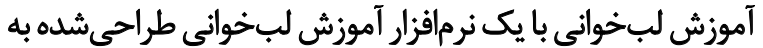

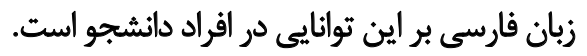

\section{مواد و روشها}

اين مطالعه به صورت مداخلهاى قبل و بعد از آموزش لبخوانى إنهان

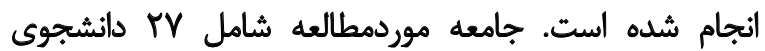

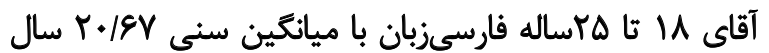

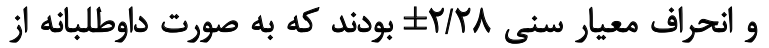

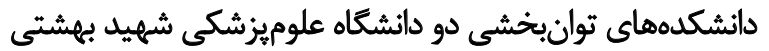

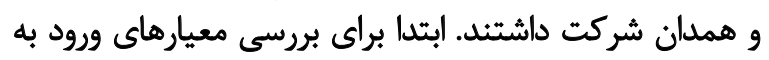

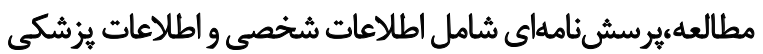

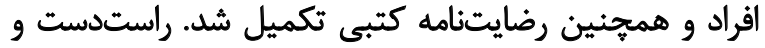

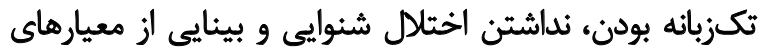

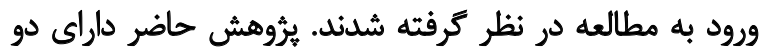

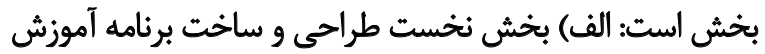
لبخوانى و شامل هفت مرحله است:

ا. انتخاب روش آموزش بر مبناي شيوههاي آموزشي رايج:

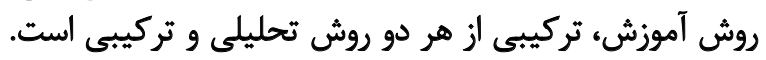

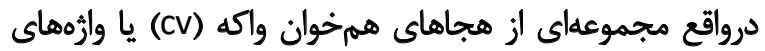

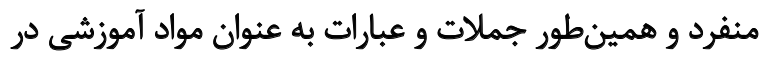
نظر كرفته شد.

ז. بررسى واكهها و همخوانهاي زبان فارسى بر اساس جايكاه

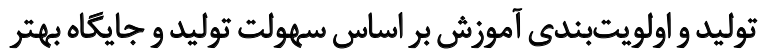

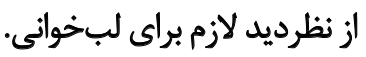

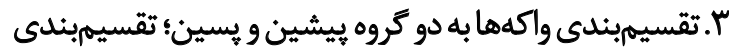

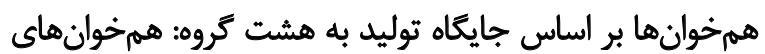

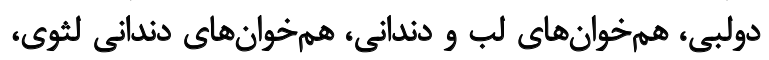

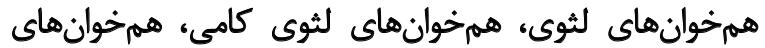
كامى، همخوان هاى ملازى، همخوان هاى جان جاكنايى.

f

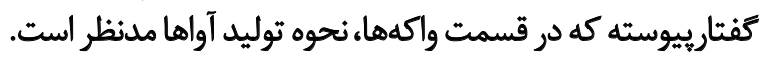

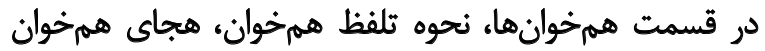

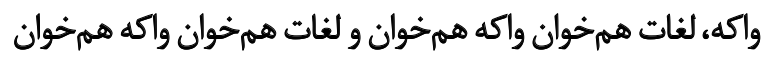

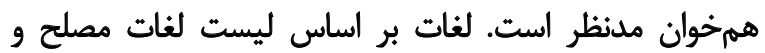

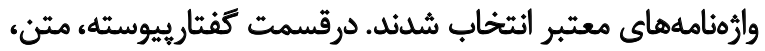

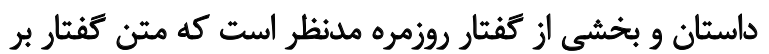

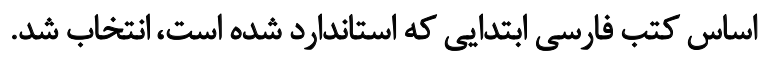
ه. هـ از تدوين مراحل قبل وارد مرحله تصويربردارى 
تصويربردارى، توسط جند نفر از استادان مجرب مورد تأييد قرار

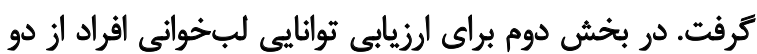
آزمون لبخوانى شماره ا و ب سارا استفاده شد.

يافتهها

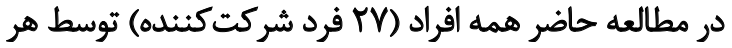

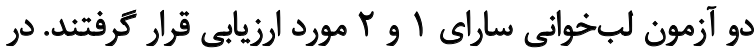

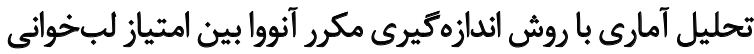

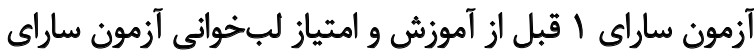

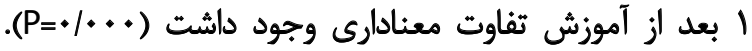

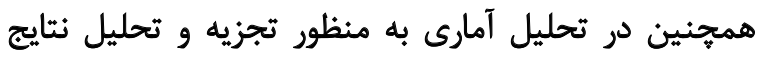

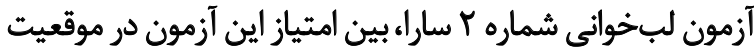

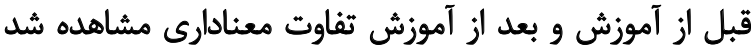
(P=×/...)

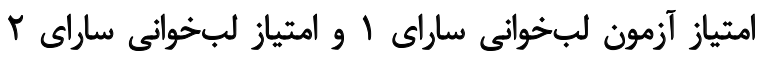

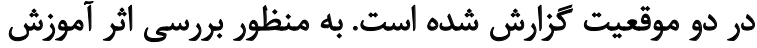

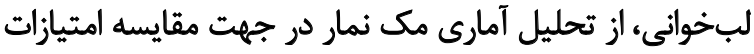

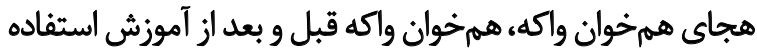

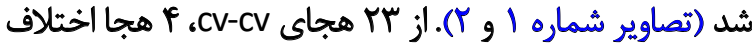

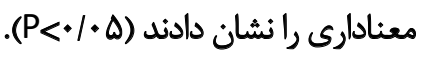

همجنين در محتواى آزمون لبخوانى ساراى اكه اكه در كل

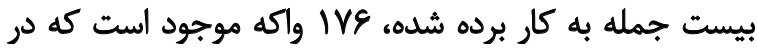

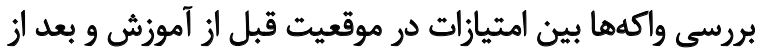

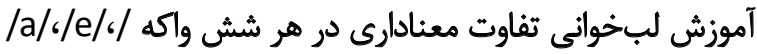

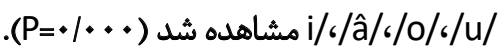

\section{بحث}

در مقايسه امتياز آزمون لبخوانى ساراى الدر دو دو موقعيت

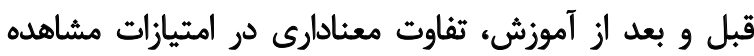

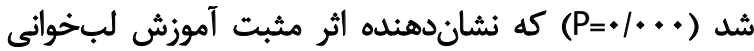

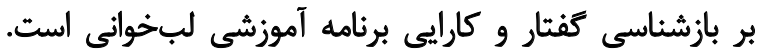
درحقيقت ميانكين درصد امتيازات بازشناسى كفيتار بهارئ وسيله
كطح نويز معمولى كمتر از ·r دسىىبل مقابل صفحه نمايش

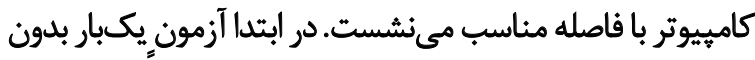

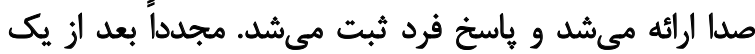

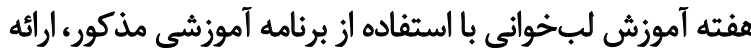

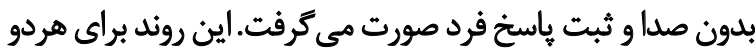

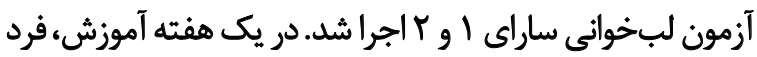

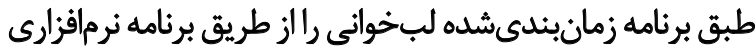

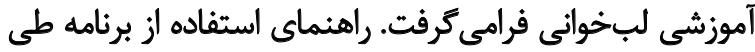

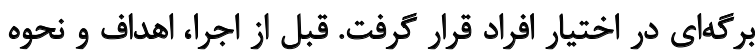

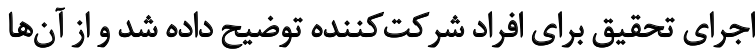

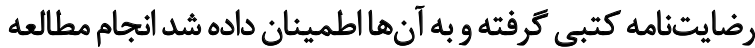

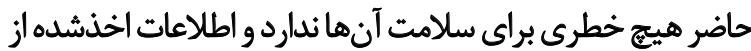

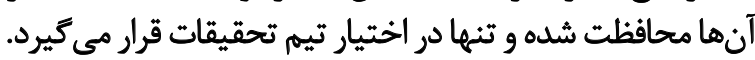
دادههاى مطالعه با استفاده از نرمافزار آمارى SPSS نسخه

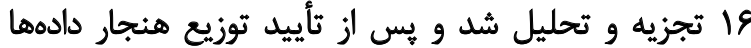

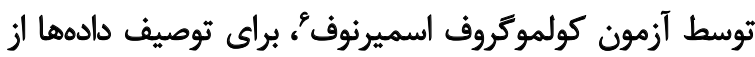

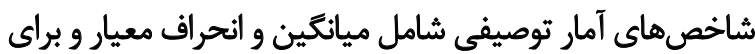

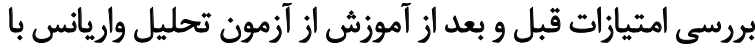

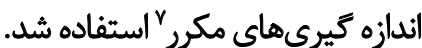

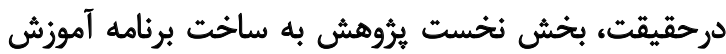

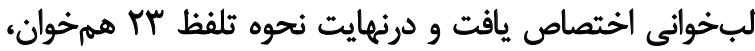

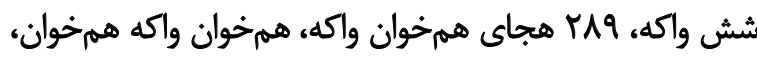

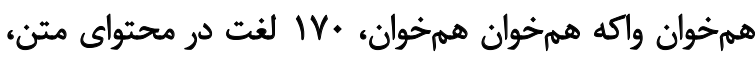

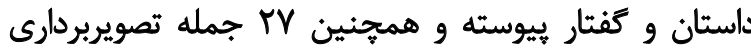

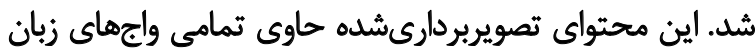

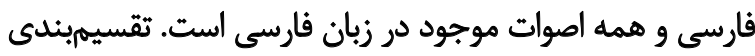

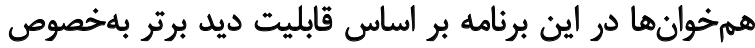

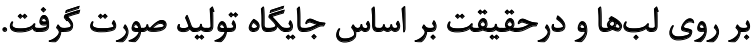

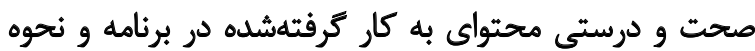

6. Kalmogorov - Smiranov

7. Repeated Measures ANOVA

CV-CV درصد امتيازات هجاي

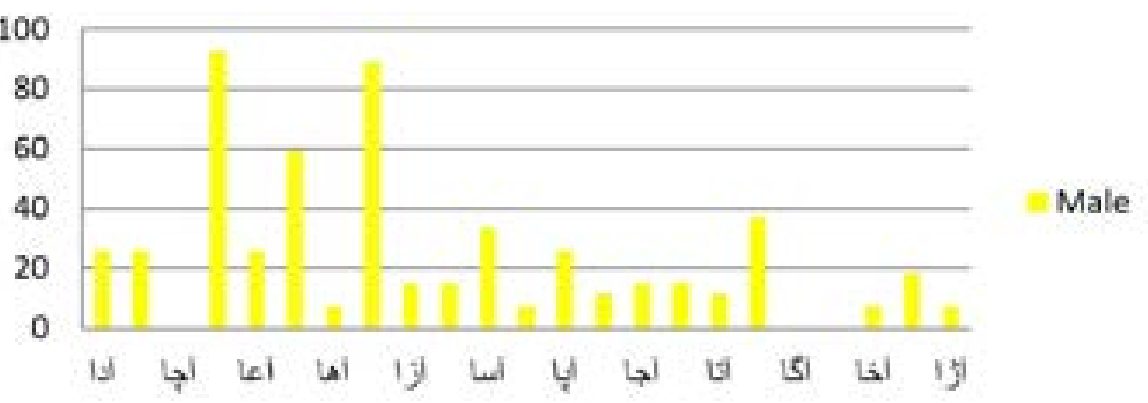


جدول ا. شاخصهاي آمارى امتيازات لبخوانى آزمون ساراي او ب در دو موقعيت قبل و بعد از آهوزش لبخوانى

\begin{tabular}{|c|c|c|c|c|c|}
\hline انحراف استاندارد & حداكثر & 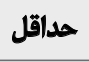 & مياتكَين & ت ت تعداد & 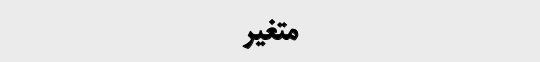 \\
\hline r/Ar & Q. & • & $10 / 19$ & Tr & امتياز آزمون لبخواني ساراى الدر موقعيت \\
\hline $4 / .1$ & A. & $\Delta$ & $F r / F i$ & TV & 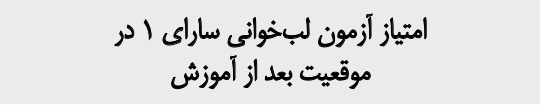 \\
\hline $1 / 81$ & $\Delta V / E$ & $1 K / 9$ & $M / F i$ & Tr & 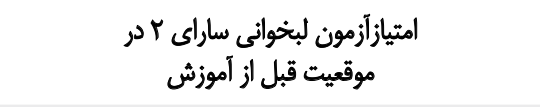 \\
\hline $1 / 8 \wedge$ & $\Delta V / 8$ & $\operatorname{lV} / \mathrm{r}$ & $r \Delta / \Delta 1$ & $r$ & امتياز آزمون لبخوانى ساراى r در موقعيت بعد از آموزش \\
\hline
\end{tabular}

آموزشهاى فردى و كروهى و آموزشهاى كودكان و سالمندان

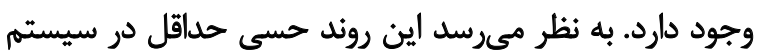

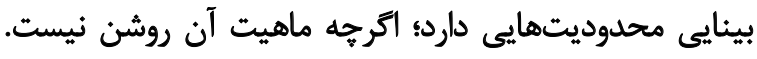

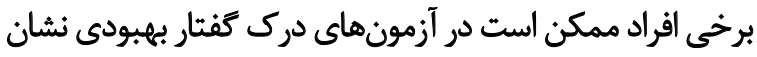

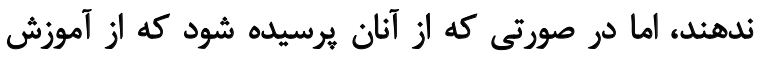

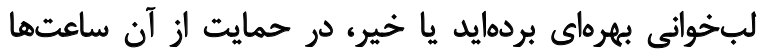

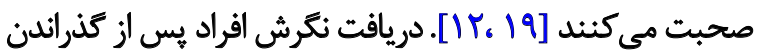

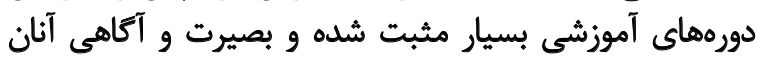

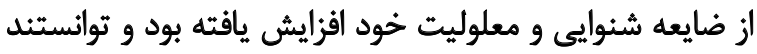
ارتباط بهترى با اطرافيان خود برقرار كثند.

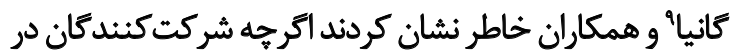

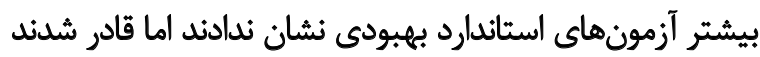

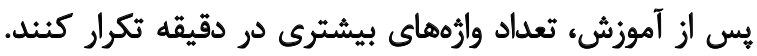

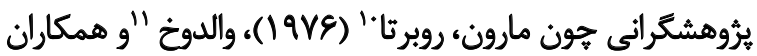

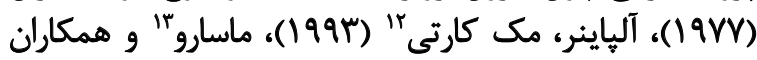
9. Gagne
10. Maron, Roberta
11. Valdokh
12. Alpiner, Mc Carthy
13. Massaro

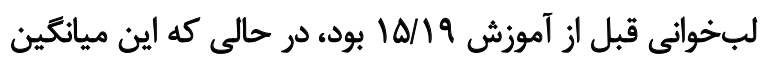

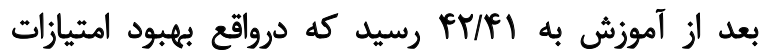

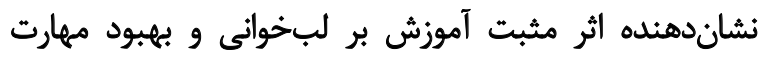

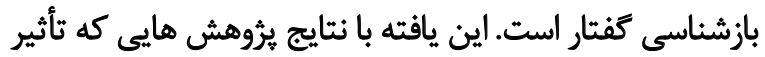

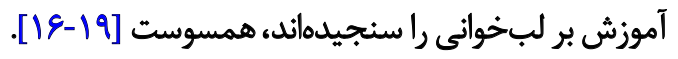

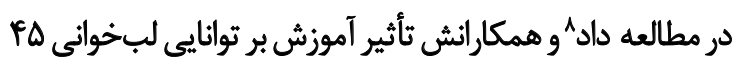

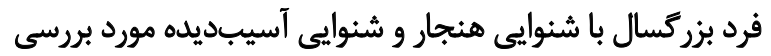

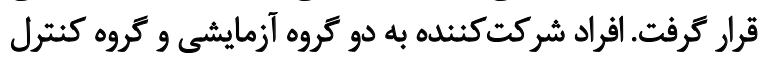

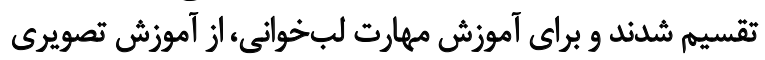

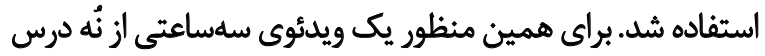

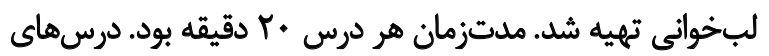

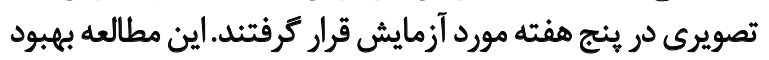

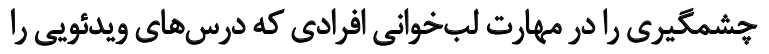

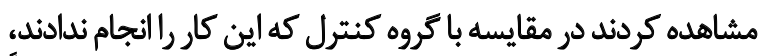

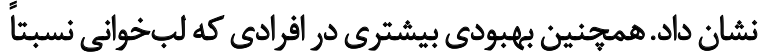

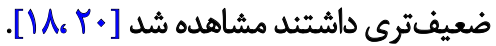

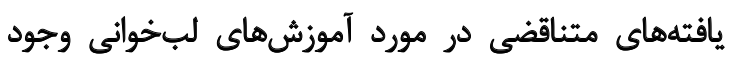

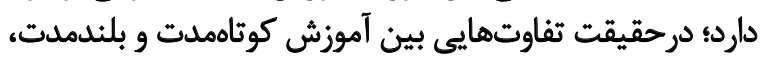

8. Dodd

\section{vرصد امتيازات هجاى CV-cV}

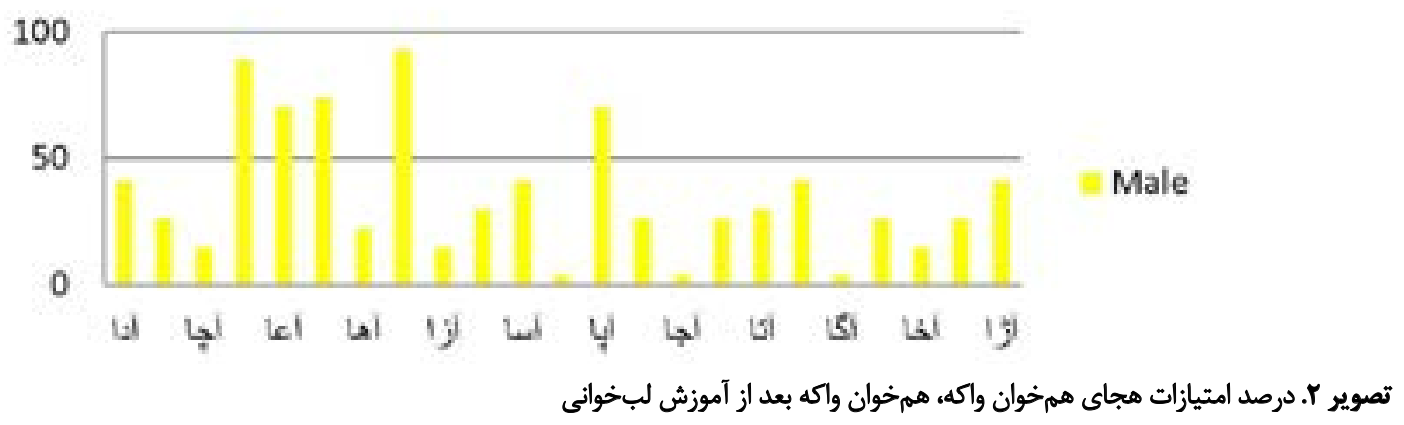


ساراى او Y و همجينين محتواى متفاوت هر دو آزمون، مى توان

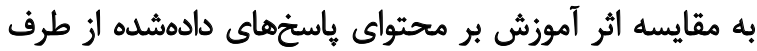

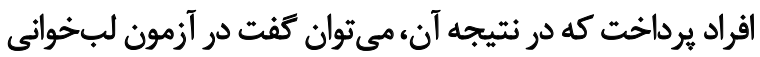

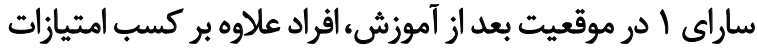

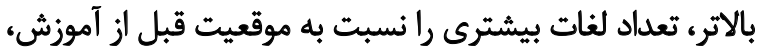

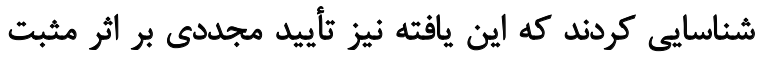

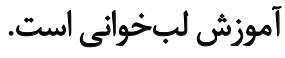

همجنين در آزمون همخوان بينواكداى سارا (آزمون ساراى

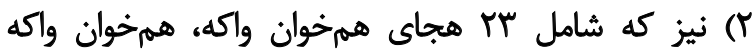

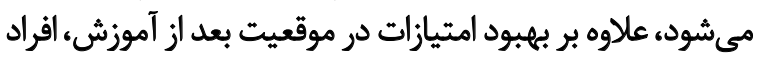

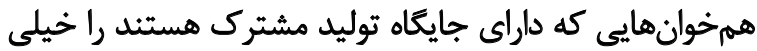

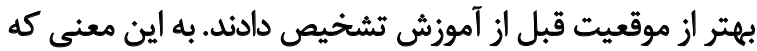

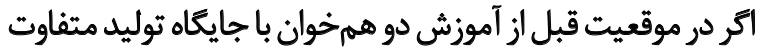

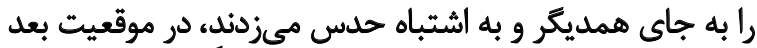

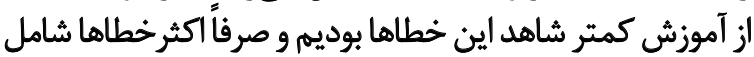

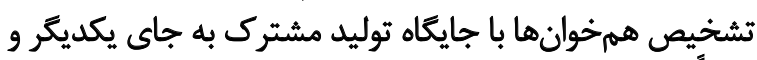

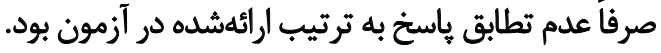

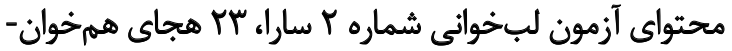

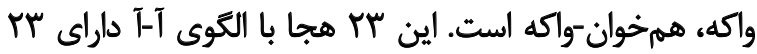

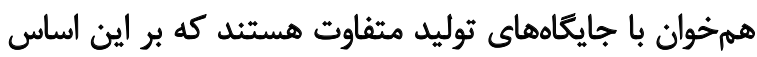

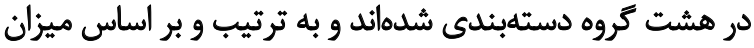

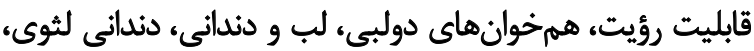

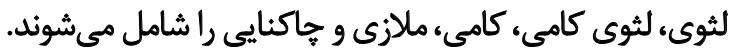
علاوه بر بررسىهاى بيشين، در اين يُؤوهش امتيازات جفت

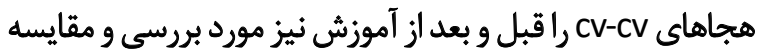

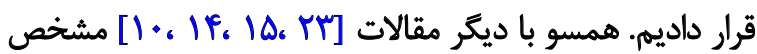

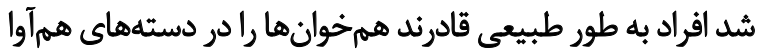

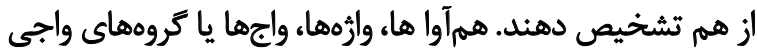

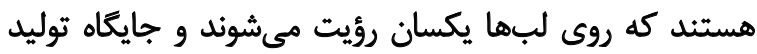

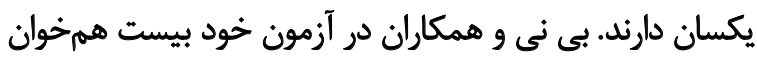

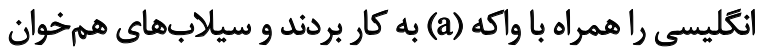

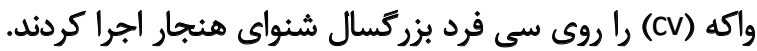

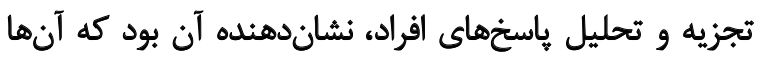

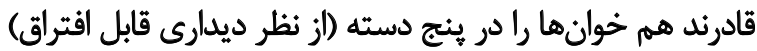

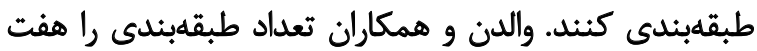

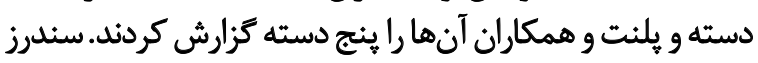

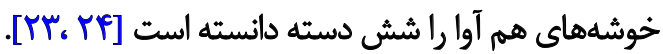
در موارد توانبخشى، تجزيه و تحليل الكوى اشتباهات فرد

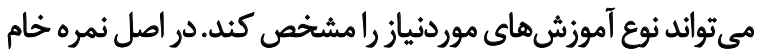

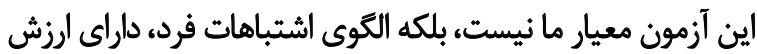

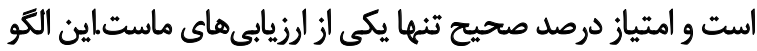

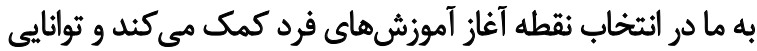

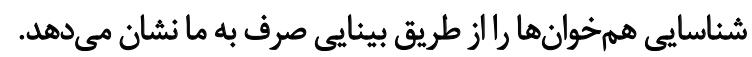

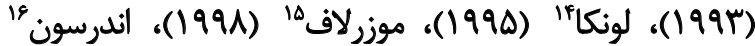

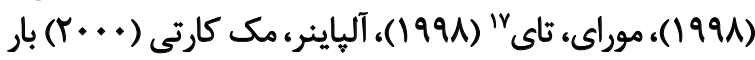

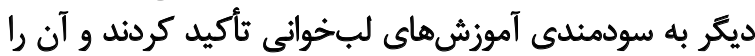

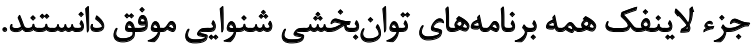

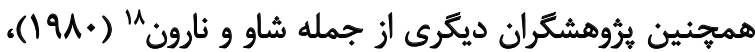

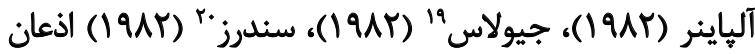

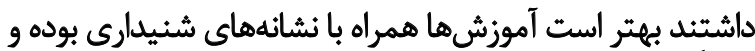

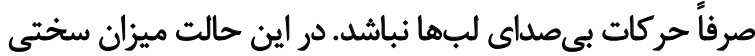

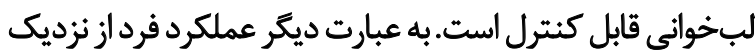

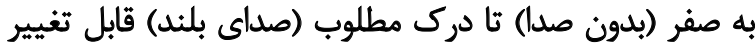

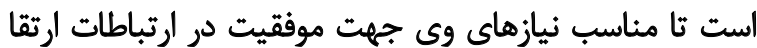

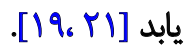

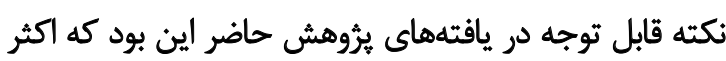

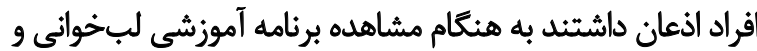

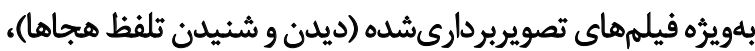

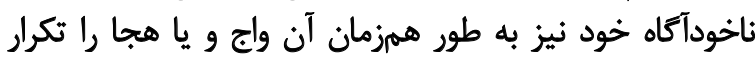

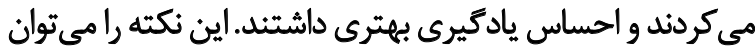

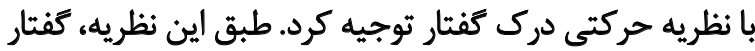

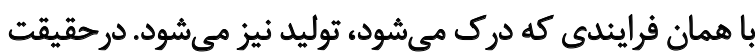

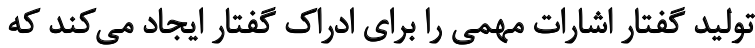

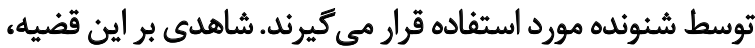

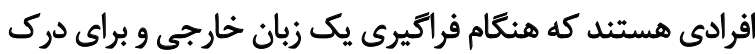

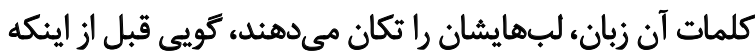

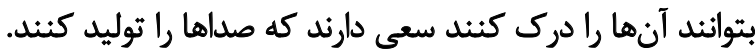

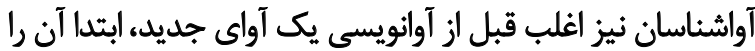

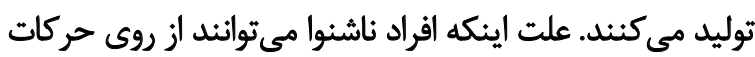

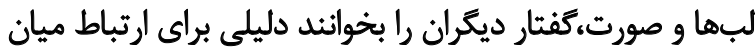

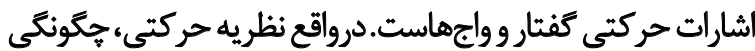

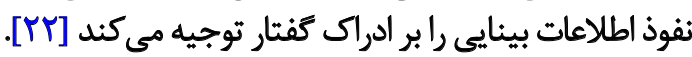

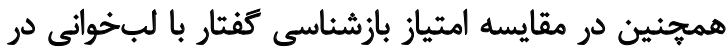

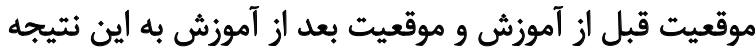

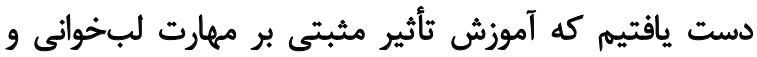

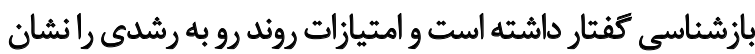
دادند؛ به طورى كه ميانكين درصد امتيازات آزمون لب آبخواني

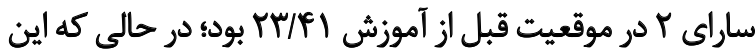

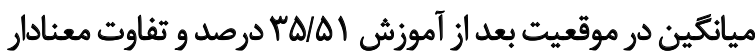

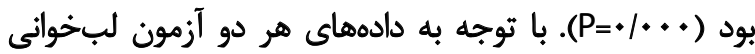

14. Lonka

15. Mozerlof

16. Anderson

17. Morray, Tay

18. Schow g Noronne

19. Giolas

20. Sanders 
اين دو واكه گرد شده و از لحاظ ديدارى توجه بيشترى را جلب

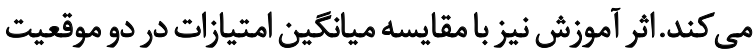

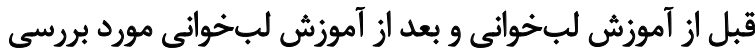

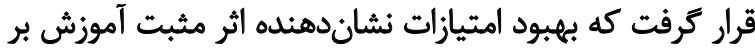

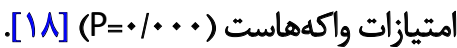

\section{تثيجلئيرى}

هدف از اين مطالعه، ساخت برنامه آموزشى لبخخوانى و بررسى

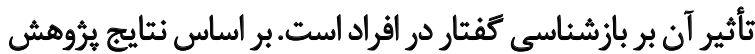

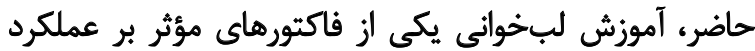

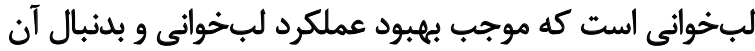

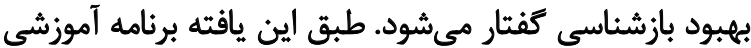

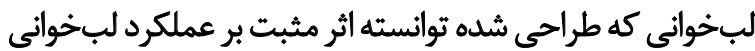

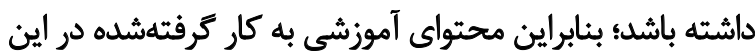

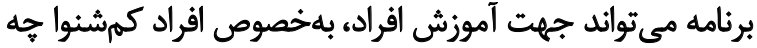

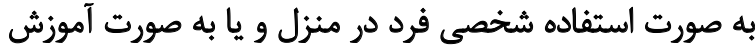

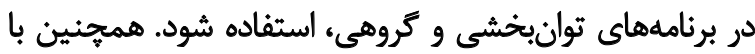

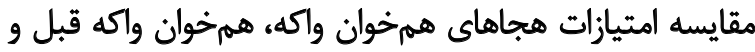

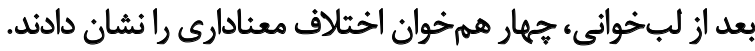

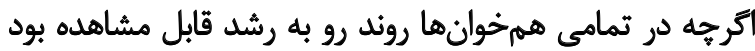

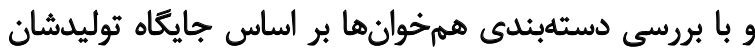

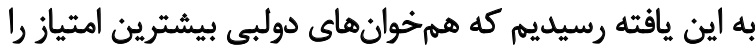

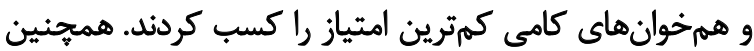

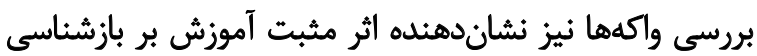

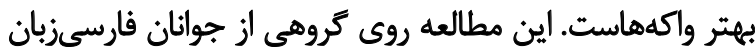

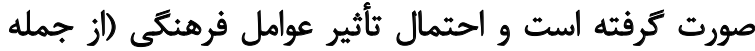

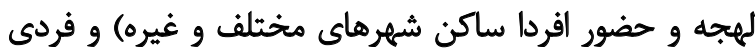

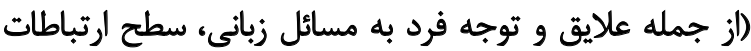

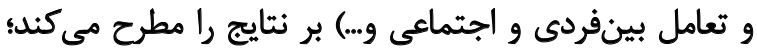

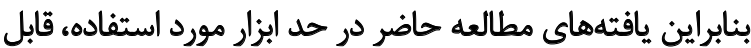

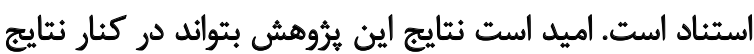

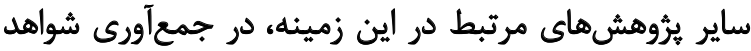

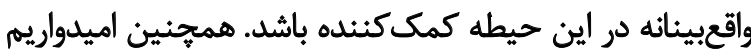

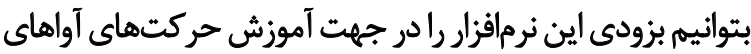

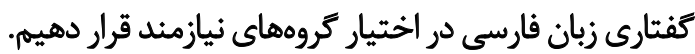

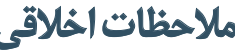

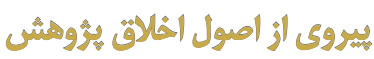

IR.SBMU. اين مقاله داراي كد اخلاق به شماره REC.1394.144 از كميته اخلاق دانشكاه علوميزشكى شهيد

$$
\text { باهي مالئ است. }
$$

با بررسى بيشتر در امتيازات هجاى CV-CV و مقايسه اين امتيازات

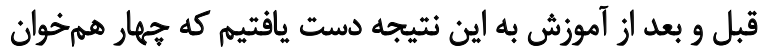

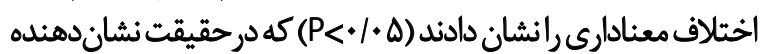

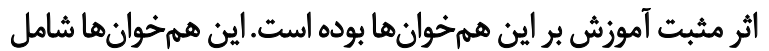

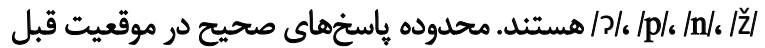

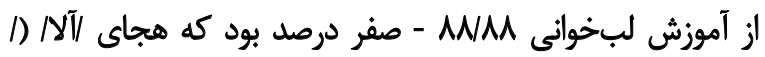

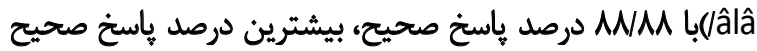

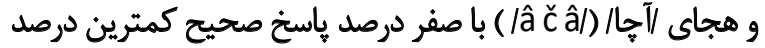

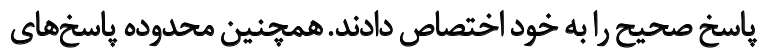

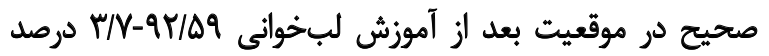

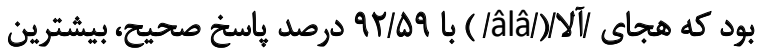

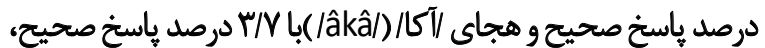

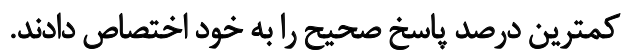

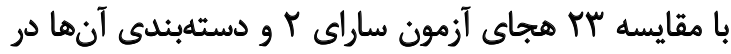

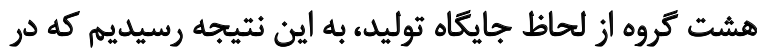

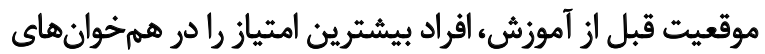

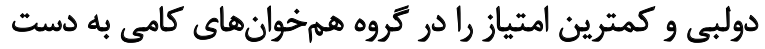

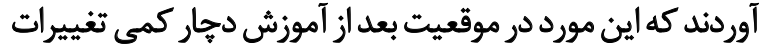

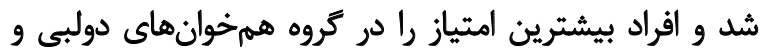

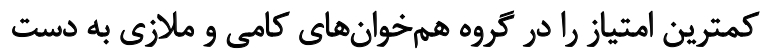

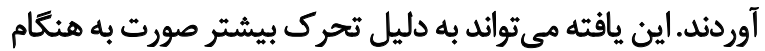

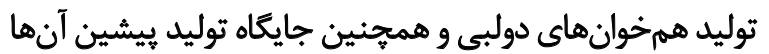

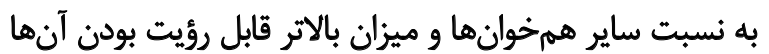

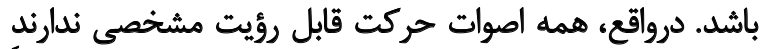

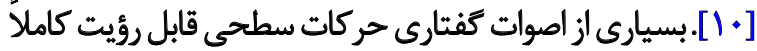

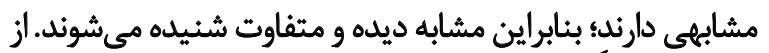

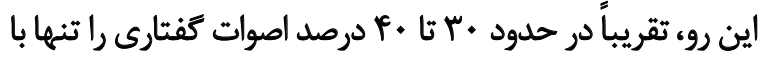
اتكا به بينايى مى توان فهميد. در محتواى آزمون لبخوانى ساراى الكه در كل بيست جمله

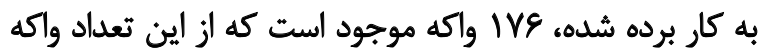

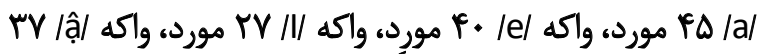

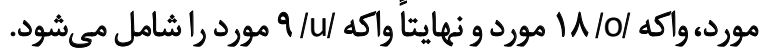

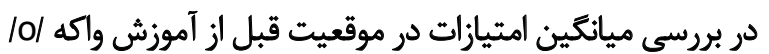

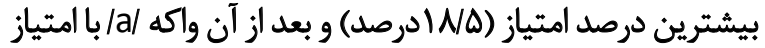

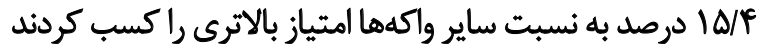

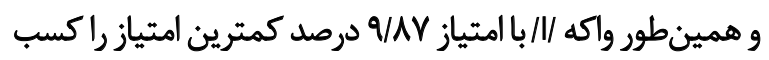

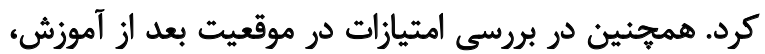

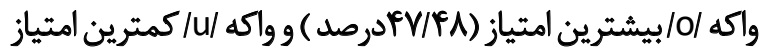

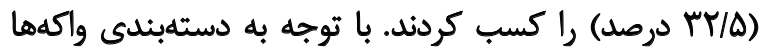

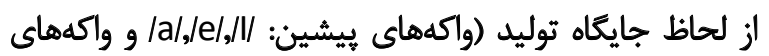

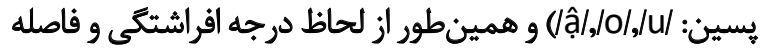

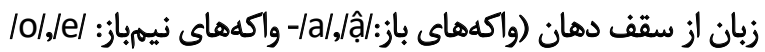

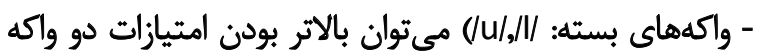

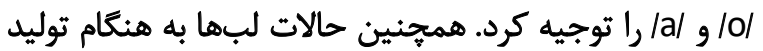


اين تحقيق هيج كمك مالى خاصى از سازمان هاي تأمين مالى دئي

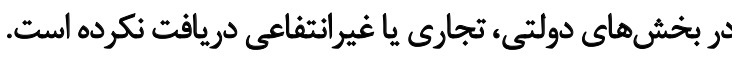

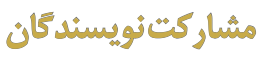

تمامى نويسندكان در آمادهازى اين مقاله به يك اندازه مشاركت داشتهاند.

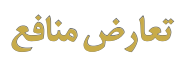

نويسند كان مقاله هيجگونه تعارضى در منافع اعلام نكردند.

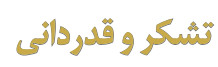

نويسندكان بر خود لازم مي دائند تا از هميارى مركز تحقيقات

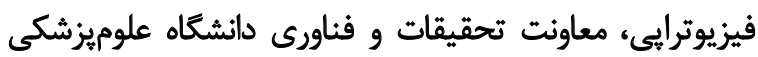

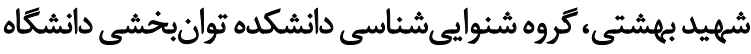

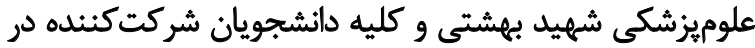

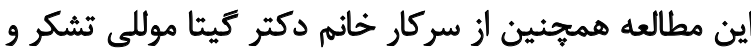
قدردانى كنيند. 


\section{References}

[1] Schwartz JL, Berthommier F, Savariaux C. Seeing to hear better: Evidence for early audio-visual interactions in speech identification. Cognition. 2004; 93(2):B69-B78. [DOI:10.1016/j.cognition.2004.01.006] [PMID]

[2] de los Reyes Rodríguez Ortiz I. Lipreading in the prelingually deaf: What makes a skilled speechreader? Span J Psychol. 2008; 11(02):488-502. [DOI:10.1017/S1138741600004492] [PMID]

[3] Deypir M, Alizadeh S, Zoughi T, Boostani R. Boosting a multi-linear classifier with application to visual lip reading. Expert Syst Appl. 2011 38(1):941-8. [DOl:10.1016/j.eswa.2010.07.078]

[4] Movallali G. [Review of half century of studies about diary speech reading (Persian)]. Audiology. 2002; 11(1):65-76.

[5] Meier U, Stiefelhagen R, Yang J, Waibel A. Towards unrestricted lip reading. Intern J Pattern Recognit Artif Intell. 2000; 14(05):571-85. [DOI:10.1142/S0218001400000374]

[6] Maidment DW, Macken B, Jones DM. Modalities of memory: Is reading lips like hearing voices? Cognition. 2013; 129(3):471-93. [DOI:10.1016/j. cognition.2013.08.017] [PMID]

[7] Feld J, Sommers M. There goes the neighborhood: Lipreading and the structure of the mental lexicon. Speech Commun. 2011; 53(2):220-8. [DOI:10.1016/j.specom.2010.09.003] [PMID] [PMCID]

[8] Mohammadzadeh A, Nureddini SZ, Sandoughdar N. [Recognition score of nasal consonants in babble noise (Persian)]. J Paramed Sci Rehabil. 2016; 5(3):34-41. [DOI:10.22038/JPSR.2016.7355]

[9] Nureddini SZ, Mohammadzadeh A, Tabatabai SM. [Comparison the recognition score of stop and fricative consonants in babble noise (Persian)]. Sci J Rehabil Med. 2015; 4(1):133-41. http://medrehab.sbmu. ac.ir/article_1100163.html

[10] Movallali G, Abdollahzadeh Rafi M. Cued speech: Full access to spoken language for the hearing impaired. Aud Vestib Res. 2012; 21(2):1-18. https://avr.tums.ac.ir/index.php/avr/article/view/455

[11] Alpiner JG, McCarthy PA. Rehabilitative audiology: Children and adults, Philadelphia: Lippincott Williams \& Wilkins; 2000.

[12] Movalleli G. [Lip reading (speech reading) instructing (Persian)]. Audiology. 2004; 13(1):45-50.

[13] Vroomen JHM. Hearing voices and seeing lips: Investigations in the psychology of lipreading [PhD. dissertation]. Brabant: Katolieke Univ; 1992.

[14] Movalleli G. [Sara lip-reading test: Construction, evaluation and operating on a group of people with hearing disorder (Persian)] [MSc. thesis]. Tehran: Tehran University of Medical Sciences; 2002.

[15] Sanders DA. Aural rehabilitation: A management model. New York City: Pearson College Div; 1982.

[16] Movallali G, Parhoun K, Daneshmandan N. [Lip reading and speech perception of hearing impaired students in special schools for the deaf in Tehran (Persian)]. Arch Rehabil. 2013; 14(2):29-37. http://rehabilitationj.uswr.ac.ir/article-1-1122-en.html

[17] Campbell R. Speechreading: Advances in understanding its cortical bases and implications for deafness and speech rehabilitation. Scand Audiol. 1998; 27(4):80-6. [DOI:10.1080/010503998420694] [PMID]
[18] Dodd B, Plant G, Gregory M. Teaching lip-reading: The efficacy of lessons on video. $\mathrm{Br} J$ Audiol. 1989; 23(3):229-38. [DOI:10.3109/03005368909076504] [PMID]

[19] Pichora-Fuller MK, Benguerel AP. The design of CAST (Computeraided speechreading training). J Speech Hear Res. 1991; 34(1):202-12. [DOI:10.1044/jshr.3401.202] [PMID]

[20] Black JW, O'Reilly PP, Peck L. Self-administered training in lipreading. J Speech Hear Disord. 1963; 28(2):183-6. [DOI:10.1044/jshd.2802.183] [PMID]

[21] Massaro DW, Cohen MM, Gesi AT. Long-term training, transfer, and retention in learning to lipread. Percept Psychophys. 1993; 53(5):54962. [DOI:10.3758/BF03205203] [PMID]

[22] Liberman AM, Cooper FS, Shankweiler DP, Studdert-Kennedy M. Perception of the speech code. Psychol Rev. 1967; 74(6):431. [DOI:10.1037/ h0020279] [PMID]

[23] Movallali G, Biglarian A. [Designing Sara Lipreading Test (No.2) and implementing it in hearing adults (Persian)]. Arch Rehabil. 2003; 4(2):53-8. http://rehabilitationj.uswr.ac.ir/article-1-611-en.htm|

[24] Hu X, Fourcin A, Faulkner A, Wei J. Speechreading of words and sentences by normally hearing and hearing impaired Chinese subjects: The enhancement effects of compound speech patterns. Speech Hear Lang Work Prog. 1996; 9:119-31. http://www.laryngograph.com/pdfdocs/ paper6.pdf 